\title{
Towards understanding the $\beta$ Pictoris dust stream
}

\author{
A. V. Krivov ${ }^{1, \star}$, N. A. Krivova ${ }^{2, \star}$, S. K. Solanki ${ }^{2}$, and V. B. Titov ${ }^{3}$ \\ 1 Institute of Physics, University of Potsdam, PO Box 601553, 14415 Potsdam, Germany \\ 2 Max-Planck-Institute for Aeronomy, Max-Planck-Strasse 2, 37191 Katlenburg-Lindau, Germany \\ 3 Astronomical Institute, St. Petersburg University, Stary Peterhof, 198504 St. Petersburg, Russia
}

Received 22 September 2003 / Accepted 26 November 2003

\begin{abstract}
The recent radar detection by Baggaley (2000) of a collimated stream of interstellar meteoroids postulated to be sourced at $\beta$ Pictoris, a nearby star with a prominent dust disk, presents a challenge to theoreticians. Two mechanisms of possible dust ejection from $\beta$ Pic have been proposed: ejection of dust by radiation pressure from comets in eccentric orbits and by gravity of a hypothetical planet in the disk. Here we re-examine observational data and reconsider theoretical scenarios, substantiating them with detailed modeling to test whether they can explain quantitatively and simultaneously the masses, speeds, and fluxes. Our analysis of the stream geometry and kinematics confirms that $\beta$ Pic is the most likely source of the stream and suggests that an intensive dust ejection phase took place $\sim 0.7 \mathrm{Myr}$ ago. Our dynamical simulations show that high ejection speeds retrieved from the observations can be explained by both planetary ejection and radiation pressure mechanisms, providing, however, several important constraints. In the planetary ejection scenario, only a "hot Jupiter"-type planet with a semimajor axis of less than $1 \mathrm{AU}$ can be responsible for the stream, and only if the disk was dynamically "heated" by a more distant massive planet. The radiation pressure scenario also requires the presence of a relatively massive planet at several AU or more, that had heated the cometesimal disk before the ejection occurred. Finally, the dust flux measured at Earth can be brought into reasonable agreement with both scenarios, provided that $\beta$ Pic's protoplanetary disk recently passed through an intensive short-lasting ( $\sim .1 \mathrm{Myr}$ ) clearance stage by nascent giant planets, similar to what took place in the early solar system.
\end{abstract}

Key words. meteors, meteoroids - stars: individual: $\beta$ Pic - stars: circumstellar matter - stars: planetary systems celestial mechanics - methods: $N$-body simulations

\section{Introduction}

After the discovery of circumstellar dust around Vega by IR excess (Blackwell et al. 1983; Harvey et al. 1984) and the first images of an extended dust disk around $\beta$ Pictoris (Smith \& Terrile 1984) it was quickly realized (Weissman 1984) that dust disks around main-sequence stars are not primordial and are signposts of cometary or asteroidal bodies. This led to a direct analogy with our solar system, the only planetary system known at that time. The question of whether planets or protoplanets exist in these systems was raised by the first detection of an extrasolar planet orbiting a main-sequence star (Mayor \& Queloz 1995). The general view that extrasolar planetary systems, like our solar system, must comprise both planets and minor body populations is now widely recognized, conforming both to observational facts and planetary formation theories (Krivova 2002).

$\beta$ Pictoris, $a \sim 10^{7}$ year-old (Ortega et al. 2002) mainsequence star with a prominent extended dust disk provides a

Send offprint requests to: A. V. Krivov, e-mail: krivov@agnld.uni-potsdam.de

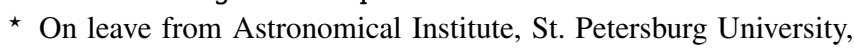
Russia. good example. It is now widely believed (Artymowicz 1997; Lecavelier des Etangs 2000) that $\beta$ Pic is most likely a planetary system at a late stage of planet formation, comprising populations of km-sized solids (planetesimals and/or cometesimals that act as a source of visible dust) and one (or several) giant planets or protoplanets (although the direct evidence for these is still lacking).

Although the $\beta$ Pic system seems to be unique in many respects among a hundred known stars with debris disks (Lecavelier des Etangs 2000), it came as a surprise when Baggaley (2000), using the Advanced Meteor Orbit Radar (AMOR) in New Zealand, detected a collimated stream of interstellar radio meteors and identified its source as $\beta$ Pic. Confirmation of the $\beta$ Pic source would offer a powerful tool for studies of other planetary systems: in situ measurements of material coming from other suns. The issue has become unclear, however, with the realization that (i) a detectable dust flux at Earth inevitably translates to a high mass loss rate at the source, and (ii) measured speeds of the stream particles at Earth translate to very high $\left(\approx 25 \mathrm{~km} \mathrm{~s}^{-1}\right)$ ejection speeds of dust grains from the source.

So far, two mechanisms have been suggested. Grün \& Landgraf (2001) have proposed a radiation pressure ejection of 
material from highly eccentric parent bodies (comets or cometesimals) in the circumstellar disk near periastra of their orbits. Krivova \& Solanki (2003) have conjectured ejection of dust by a nascent planet in the $\beta$ Pictoris disk. Note that the radiation pressure scenario is not "planet-free" either: a planet is required to force parent bodies of the ejected dust into nearly star-grazing orbits. Furthermore, a planet is needed anyway to explain observed falling-evaporating bodies (FEBs), introduced as an interpretation of frequent, transient, mostly redshifted spectral events observed for many years (Lagrange et al. 1987; Beust et al. 1989, 1991, 1996; Beust \& Morbidelli 1996, 2000; Thébault \& Beust 2001).

The goal of this paper is to reconsider both the observational data and the theoretical scenarios in an attempt to explain the phenomenon. In Sect. 2, observational data about the meteoroid stream and the $\beta$ Pic system are discussed. Then we consider the "speed" problem, checking both the planetary ejection (Sect. 3) and the radiation pressure ejection scenarios (Sect. 4) and finding constraints on the presumed planet(s) and dust parent bodies in the $\beta$ Pic system. Section 5 addresses the "flux problem". Our conclusions are presented in Sect. 6.

\section{Observational data and their interpretation}

\subsection{Dust stream}

\section{Observational data}

The following properties of the stream have been derived (Baggaley 2000; Baggaley \& Galligan 2001): (i) the dust particles are large, with masses $\gtrsim 10^{-7} \mathrm{~g}$ or radii $\gtrsim 20 \mu \mathrm{m}$. (ii) Analysis of heliocentric velocities and local stellar kinematics implies ejection from the $\beta$ Pictoris system at speeds $\approx 25 \mathrm{~km} \mathrm{~s}^{-1}$, so that, for a distance to $\beta$ Pic of $19.3 \mathrm{pc}$ (Crifo et al. 1997), they must have left the system about 1 Myr ago. (iii) The flux is hard to quantify, given the uncertainties in the absolute radar detection calibration arising from the plasma creation dependence on meteoroid speed and mass, corrections for reflection process and the radar response function. Based upon $\sim 3$ detections per day (which is an upper limit suggested by the data available for several years) and an effective radar collecting area of $120 \mathrm{~km}^{2}$ for the radar antenna configuration (Baggaley, pers. comm.), the estimated flux at Earth is $\sim 3 \times 10^{-17} \mathrm{~cm}^{-2} \mathrm{~s}^{-1}$. Of course, this value refers to particles with radii above $\approx 20 \mu \mathrm{m}$, which fall into the sensitivity range of the radar.

\section{Identification of the source}

We now check the conclusion that $\beta$ Pic is the likely source of the meteoroid stream on the basis of stellar and dust kinematics calculations. The idea is to write down and solve simple equations of dust propagation between a candidate star and the Sun and to check whether that star is compatible with being the source of the observed stream.

Consider the candidate star, the Sun, and a dust grain (Fig. 1). Kinematical quantities related to them will be marked by subscripts $\mathrm{B}, \mathrm{S}$ and $\mathrm{D}$, respectively. To distinguish between the moment of dust ejection $t_{0}$ and the moment of

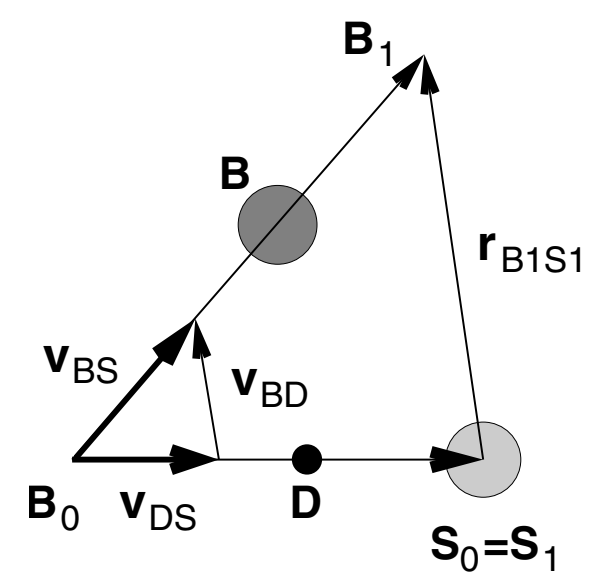

Fig. 1. Geometry of propagation of dust (D) between the source star (B) and the Sun (S) in the heliocentric reference frame. The symbols are explained in the text.

observation (i.e., the present time) $t_{1}$ we will add " 0 " and " 1 " to these subscripts, when necessary. The radius vector and velocity of an object "P" relative to object "Q" will be denoted by $\boldsymbol{r}_{\mathrm{PQ}}$ and $\boldsymbol{v}_{\mathrm{PQ}}$. We assume a rectilinear motion of $\mathrm{B}, \mathrm{S}$, and $\mathrm{D}$ with a constant speed. This is a good approximation for B and $\mathrm{S}$ on timescales of $\lesssim 1$ Myr (Ortega et al. 2002) as well as for the dust particles. Indeed, the dust grains, owing to their relatively large sizes, are not subject to any non-gravitational forces (Baggaley $\&$ Neslušan 2002). The gravity of other stars is not likely to bend the dust grain trajectories either. To deflect a grain trajectory by several degrees, a star of a solar mass should pass by the grain during its flight from $\beta$ Pic at a distance of $\approx 20$ AU. Given the stellar density in the neighborhood of the Sun of $0.1 \mathrm{pc}^{-3}$, typical stellar velocities of $30 \mathrm{~km} \mathrm{~s}^{-1}$, and the propagation time of the dust stream of $\sim 1 \mathrm{Myr}$, the probability of such an event is about $10^{-7}$. The equations of motion of the objects give:

$\boldsymbol{r}_{\mathrm{B} 1 \mathrm{~S} 1}=\left[\boldsymbol{v}_{\mathrm{BS}}-\boldsymbol{v}_{\mathrm{DS}}\right] \Delta t$,

where $\Delta t=t_{1}-t_{0}$. Note that our method is different from that of Baggaley (2000). Instead of considering the motion of the candidate stars and the Sun with respect to the LSR which are not free of large uncertainties, we use $U, V, W$ components of the star's velocity with respect to the Sun, which are direct observables: $\boldsymbol{v}_{\mathrm{BS}} \equiv(U, V, W)$. The $\sigma$ in each component is typically $2 \mathrm{~km} \mathrm{~s}^{-1}$, so we independently vary all three within a $1 \sigma$ range when solving the equations. We also use the observed location (uncertainty $\approx 3^{\circ}$ ) of the centroid of the far-Sun upstream inflow directions (solar gravity removed) on the celestial sphere: ecliptic latitude $-56^{\circ}$ and longitude $280^{\circ}$ and observed angular size about $20^{\circ}$, as reported by Baggaley (2000). Next, the heliocentric entry speed of the particles derived from the observations, from 9 to $15 \mathrm{~km} \mathrm{~s}^{-1}$, is used as input. Both the location of the meteoroid "spot" on the celestial sphere and the heliocentric speed of the meteoroids determine the velocity vector $\boldsymbol{v}_{\mathrm{DS}}$ in Eq. (1). Finally, we calculate $\boldsymbol{r}_{\mathrm{B} 1 \mathrm{~S} 1}$ in the same equation from the right ascension and declination of the candidate star and a distance to it, as given in catalogues.

Equation (1) shows that a candidate star is compatible with being the source of the stream if and only if the vector $\boldsymbol{r}_{\mathrm{B} 1 \mathrm{~S} 1}$ 
Table 1. Compatibility of selected stars with being the source of the stream.

\begin{tabular}{|c|c|c|}
\hline Star & \multicolumn{2}{|c|}{ "Compatibility" angle, deg } \\
\hline \multicolumn{3}{|c|}{$\beta$ Pic } \\
\hline$\beta$ Pic & & 0.0 \\
\hline \multicolumn{3}{|c|}{ A "field" star } \\
\hline$\alpha$ Pic & & 24.1 \\
\hline \multicolumn{3}{|c|}{ Some stars with debris disks } \\
\hline$\alpha$ PsA & & 42.6 \\
\hline$\epsilon$ Eri & & 37.5 \\
\hline$\alpha$ Lyr & & 137.2 \\
\hline \multicolumn{3}{|c|}{$B P M G$} \\
\hline HD 35850 & & 23.4 \\
\hline AO Men & & 9.1 \\
\hline V343 Nor & & 57.9 \\
\hline HR 6070 & & 92.8 \\
\hline HD 172555 & & 52.0 \\
\hline GJ 803 & & 74.1 \\
\hline
\end{tabular}

is parallel to the vector $\boldsymbol{v}_{\mathrm{BD}} \equiv \boldsymbol{v}_{\mathrm{BS}}-\boldsymbol{v}_{\mathrm{DS}}$. Thus the angle between the two can be used as a measure of compatibility of the star with being the source. We have checked: (i) $\alpha$ Pic (a star close to $\beta$ Pic on the celestial sphere); (ii) several stars with debris disks; (iii) some members of the recently discovered Beta Pictoris Moving Group (BPMG, see Sect. 2.2). Importantly, for the BPMG stars (including $\beta$ Pic) we used up-to-date values of distance and velocity components, based on PPM and Hipparcos catalogues (see Zuckerman et al. 2001). For $\alpha$ Pic and the stars with debris disks, we used the "Preliminary Version of the Third Catalogue of Nearby Stars" by Gliese \& Jahreiss (Astron. Rechen-Institut, Heidelberg, 1991) available at http://vizier.u-strasbg.fr.

The results are shown in Table 1 . For each star in our selection, it provides the best value of the "compatibility" angle, obtained by varying the star's $U, V, W$ and the heliocentric entry speed of the meteoroids as described above. The smaller the angle, the higher the compatibility of the star being the source of the dust stream. The conclusion is unambiguous: of all the stars checked, only $\beta$ Pic is consistent with the dynamics. The best value of the "compatibility" angle for $\beta$ Pic is $0.00254^{\circ}$, much smaller than the precision of the AMOR observations (several degrees). Even the value obtained without varying the parameters, i.e. just assuming the nominal $U, V, W$ and the heliocentric entry speed of $11 \mathrm{~km} \mathrm{~s}^{-1}$, falls within the observational uncertainty: $2.1^{\circ}$.

The solution of the same equations for $\beta$ Pic simultaneously gave other quantities of interest: most notably, the ejection speed of the meteoroids $v_{\mathrm{DB}}$, their propagation time $\Delta t$, and the position of the star with respect to the Sun at the moment

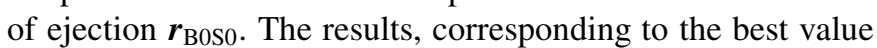
of the compatibility angle, are listed in Table 2.

For any realistic mechanism of dust ejection from $\beta$ Pic, one should expect a dispersion of ejection velocities $\boldsymbol{v}_{\mathrm{DB}}$ (or $\boldsymbol{v}_{\mathrm{DS}}$ ). This means that dust particles currently detected by the radar
Table 2. Kinematic results for the stream with $\beta$ Pic as the source. Input data used: distance to $\beta$ Pic, 19.3 pc (Crifo et al. 1997); equatorial coordinates of $\beta \mathrm{Pic}, \mathrm{Dec}=-51.1^{\circ}$ and $\mathrm{RA}=86.8^{\circ}$ (Zuckerman et al. 2001); galactic velocity components of $\beta$ Pic relative to the Sun, $(-10.8,-16.4,-8.9) \mathrm{km} \mathrm{s}^{-1}$ with $\sigma=2 \mathrm{~km} \mathrm{~s}^{-1}$ in each component (Zuckerman et al. 2001); heliocentric entry speed of meteoroids, 9 to $15 \mathrm{~km} \mathrm{~s}^{-1}$ (Baggaley 2000); ecliptic coordinates of the meteoroid "spot", LAT $=-56^{\circ}$ and $\mathrm{LON}=280^{\circ}$ (Baggaley 2000).

\begin{tabular}{|c|c|c|c|}
\hline \multicolumn{4}{|c|}{ Current heliocentric position of the star } \\
\hline distance $(\mathrm{pc})$ & 19.3 & & \\
\hline galactic LAT, LON (deg) & -30.3 & 258.4 & \\
\hline ecliptic LAT, LON (deg) & -74.4 & 82.4 & \\
\hline galactic $x, y, z(\mathrm{pc})$ & -3.4 & -16.3 & -9.7 \\
\hline ecliptic $x, y, z(\mathrm{pc})$ & 0.7 & 5.1 & -18.6 \\
\hline \multicolumn{4}{|c|}{ Heliocentric velocity of the star } \\
\hline modulus $\left(\mathrm{km} \mathrm{s}^{-1}\right)$ & 19.8 & & \\
\hline $\operatorname{radial}\left(\mathrm{km} \mathrm{s}^{-1}\right)$ & 18.1 & & \\
\hline galactic LAT, LON (deg) & -21.6 & 233.4 & \\
\hline ecliptic LAT, LON (deg) & -50.6 & 90.7 & \\
\hline galactic $v x, v y, v z\left(\mathrm{~km} \mathrm{~s}^{-1}\right)$ & -11.0 & -14.8 & -7.3 \\
\hline ecliptic $v x, v y, v z\left(\mathrm{~km} \mathrm{~s}^{-1}\right)$ & -0.1 & 12.6 & -15.3 \\
\hline \multicolumn{4}{|c|}{ Heliocentric velocity of dust grains } \\
\hline modulus $\left(\mathrm{km} \mathrm{s}^{-1}\right)$ & 10.7 & & \\
\hline ecliptic LAT, LON (deg) & 56.0 & 100.0 & \\
\hline ecliptic $v x, v y, v z\left(\mathrm{~km} \mathrm{~s}^{-1}\right)$ & -1.0 & 5.9 & 8.9 \\
\hline \multicolumn{4}{|c|}{ Ejection and propagation of dust } \\
\hline “compatibility” angle (deg) & 0.0 & & \\
\hline ejection speed at the source $\left(\mathrm{km} \mathrm{s}^{-1}\right)$ & 25.1 & & \\
\hline propagation time (Myr) & 0.75 & & \\
\hline \multicolumn{4}{|c|}{ Heliocentric position of the star at ejection } \\
\hline distance $(\mathrm{pc})$ & 8.2 & & \\
\hline ecliptic LAT, LON (deg) & -56.0 & 280.0 & \\
\hline
\end{tabular}

were ejected at somewhat different velocities and therefore at moments of time, differing by a certain $\delta t$. If the velocity vectors $\boldsymbol{v}_{\mathrm{BS}}$ and $\boldsymbol{v}_{\mathrm{DS}}$ are not collinear (which in the case of $\beta$ Pic they are not), this should lead to an "aberration" effect: grains with different speeds must be reaching the observer from different directions. In other words, the "dust spot" on the sky should be somewhat elongated along the path of the star on the celestial sphere at the period of ejection. Differentiating Eq. (1) and assuming $\delta t \ll \Delta t$, it is easy to get an estimate of the angular extent of the dust spot:

$\gamma \approx \frac{\delta t}{\Delta t} \frac{v_{\mathrm{BS}}}{v_{\mathrm{DS}}} \sin \left(\boldsymbol{v}_{\mathrm{BS}}, \boldsymbol{v}_{\mathrm{DS}}\right)$

whence, substituting numerical values from Table 2 ,

$\gamma \approx 100^{\circ} \frac{\delta t}{\Delta t}$

Figure 2 presents a "radio meteoroid map" on the celestial sphere. On the background, we show a contour plot of the ratio of the fluxes of meteoroids in open and closed orbits, detected by AMOR and discussed in detail in Baggaley (2000). A bright white spot in the right bottom part of the figure corresponds to 


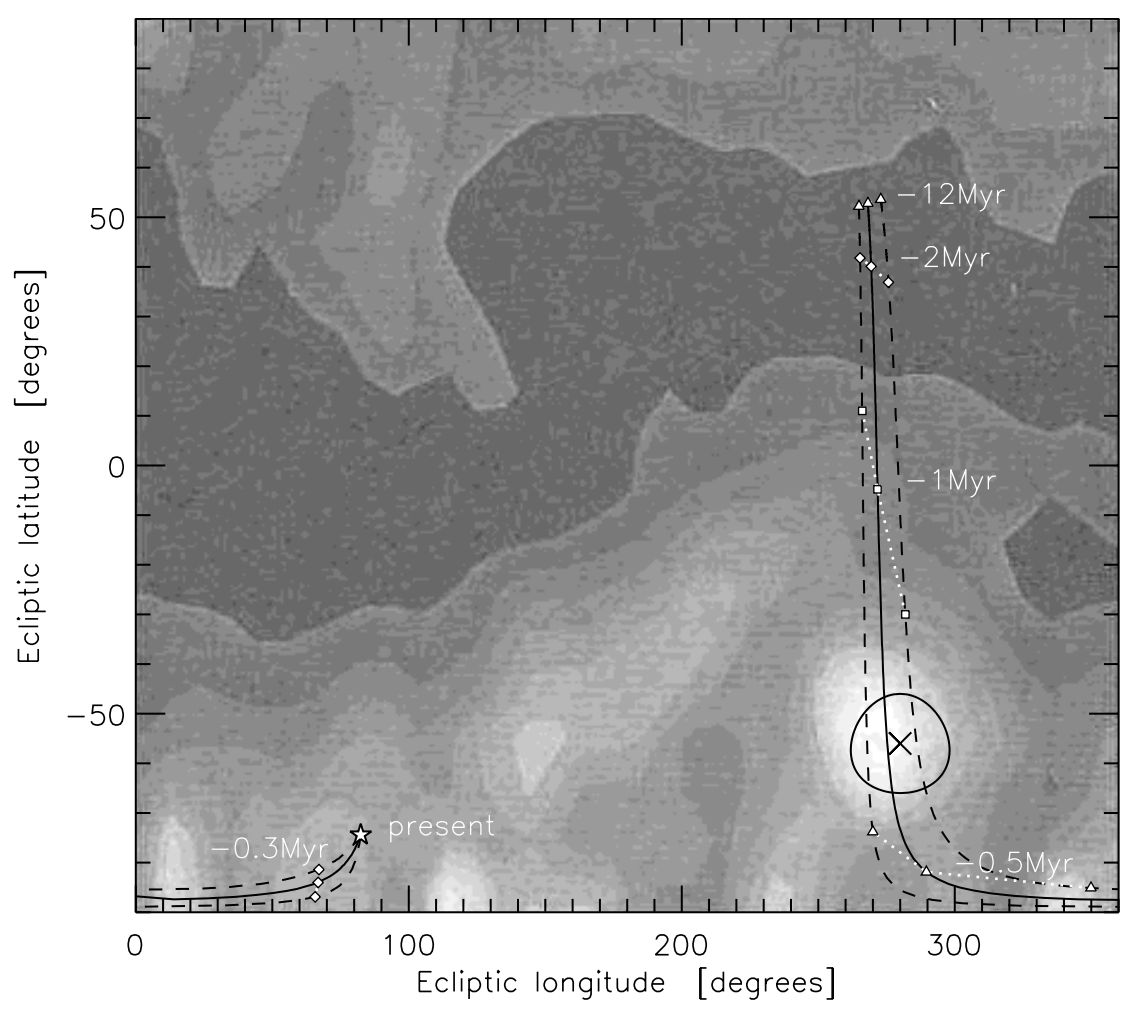

Fig. 2. The ratio of the fluxes of meteoroids in hyperbolic and closed orbits over the sky (Baggaley 2000) and the trajectory of $\beta$ Pic on the celestial sphere. The bright white spot at the bottom right is the " $\beta$ Pic dust stream". The cross marks the location of the "brightness" maximum. The closed oval around the cross consists of points which are $10^{\circ}$ away from the cross. A comparison of the oval and the spot suggests that the spot appears somewhat elliptic. The solid line depicts the path of $\beta$ Pic on the sky from its present position (marked with a star) back in time to its probable birthplace about $12 \mathrm{Myr}$ ago, with intermediate time stamps. Two dashed lines correspond to a $1 \sigma$ uncertainty. The direction of the long axis of the meteoroid spot is approximately aligned with the direction of $\beta$ Pic's motion.

a compact area on the sky where many more interstellar meteoroids are detected than interplanetary ones; it is the $\beta$ Pic dust stream under study. The best-fit location of the radiant, $\left(-56^{\circ}, 280^{\circ}\right)$, is marked with a cross. A closed oval-shaped curve around the cross is a geometrical place of points on the celestial sphere whose angular separation from the cross is $10^{\circ}$, close to the precision of AMOR observations and data reduction. In terms of spherical geometry, this curve is a small circle on a sphere, but it is not a perfect circle in the figure, which is an effect of projecting a sphere onto a plane. This oval can be used to visually judge whether or not the meteoroid spot is elongated. A comparison suggests that the spot does appear elongated from bottom to top (and slightly from the right to the left). We now check whether the ellipticity of the spot may be attributed to the aberration effect discussed above. For this purpose, we overplot the trajectory of $\beta$ Pic from its present location (indicated by a small star) backward in time. The solid line represents the trajectory calculated with the "nominal" $U$, $V, W$ of $\beta$ Pic, while two dashed lines around it approximately correspond to a $2 \mathrm{~km} \mathrm{~s}^{-1}$ uncertainty in $U, V, W$, so that the true path of the star most likely lies somewhere between the two dashed lines. Also shown are time stamps, identifying the position of the star $0.3,0.5,1,2$ and $12 \mathrm{Myr}$ ago, the last value being close to the age of $\beta$ Pic (see Sect. 2.2). As expected, the star crossed the position of the spot about $0.75 \mathrm{Myr}$ ago, which demonstrates again that $\beta \mathrm{Pic}$ is compatible with the source of the dust stream. What is more, the direction in which the spot is stretched does not differ much from the direction along the trajectory of the star. If this is caused by the aberration effect, then we can compare the extent of the spot with the time stamps on the trajectory of the star in the figure. We find that the upper edge of the spot would correspond to grains ejected $\approx 0.85 \mathrm{Myr}$ ago at a speed of $\approx 22 \mathrm{~km} \mathrm{~s}^{-1}$ and the lower edge to particles that left the $\beta$ Pic system $\approx 0.65 \mathrm{Myr}$ ago with a speed of $\approx 29 \mathrm{~km} \mathrm{~s}^{-1}$. Thus a duration of the ejection period that corresponds to the observed spot is $\delta t \sim 0.2 \mathrm{Myr}$. Approximately the same result follows from Eq. (3) with $\Delta t \sim 0.75 \mathrm{Myr}$ and $\gamma \sim 20^{\circ}: \delta t \sim 0.15 \mathrm{Myr}$.

Obviously, the precision of the AMOR measurements at present is not high enough to reliably prescribe a slight stretch of the spot to the aberration effect. Nevertheless, our consideration does set an important constraint on the system's past: we can conclude that the ejection phase in the $\beta$ Pic system did not start earlier than $\approx 0.85 \mathrm{Myr}$ ago. And indeed, for any realistic mechanism of dust ejection, the ejection speed distribution should have a negative slope: the higher the ejection speed, the less the flux of the ejected particles. In other words, many more particles slower than those responsible for the observed stream are expected. Therefore, did the ejection start earlier, the meteoroid spot would be much more extended vertically and would have a pronounced brightness gradient towards higher ecliptic latitudes. For instance, if the ejection process were at 
work as early as $\sim 1-2 \mathrm{Myr}$ ago, then (presumably more abundant) particles ejected at speeds of $\sim 10-20 \mathrm{~km} \mathrm{~s}^{-1}$ would now be arriving at the Earth and creating a bright spot between the ecliptic latitudes $-20^{\circ}$ and $+30^{\circ}$, which is not observed (see Fig. 2). Some caution is required, however, since the hyperbolic dust component near the ecliptic would be difficult to separate from the overwhelming population of closed-orbit interplanetary meteoroids, strongly concentrated towards the ecliptic (given the inherent $\sim 5 \%$ uncertainty in radar meteor speed determination). In any case, very early ejection ( $>2 \mathrm{Myr}$ ago) can be ruled out with full certainty.

Unfortunately, using the same line of reasoning we cannot constrain the duration of the ejection phase. If the ejection process was still working, say, $0.5 \mathrm{Myr}$ ago, then only very fast grains ejected at $\approx 38 \mathrm{~km} \mathrm{~s}^{-1}$ would have enough time to reach the Earth, having heliocentric entry speeds of $\approx 21 \mathrm{~km} \mathrm{~s}^{-1}$ - the slower ones would still be on the way to Earth. One may think of an additional observational test: it would be interesting to analyze a distribution of a (scarce) population of very fast meteoroids detected by AMOR to see if some of them are indeed in the lower right portion of Fig. 2. A positive result would indicate a longer ejection phase. A negative result, however, would be inconclusive - either the ejection phase was short-lasting or the ejection speed distribution is so steep that a population of fast particles is too scarce. In Sect. 5, we will show that a short duration of the ejection phase, $\sim 0.1 \mathrm{Myr}$, is favored by the analysis of the observed flux of $\beta$ Pic meteoroids. Of course, the above discussion of the ejection phase duration only applies to particles large enough to be detected by AMOR. It is possible that the $\beta$ Pic system, as every other (proto)planetary system, continuously loses smaller grains resulting from collisions and evaporation of cometesimals.

As noted above, the location of the meteoroid source on the celestial sphere was deduced by Baggaley (2000) after applying a correction for the gravitational bending of the grain trajectories by the gravity of the Sun. It is known, however, that for any collimated stream of interstellar particles penetrating the solar system and at any moment in time, there are two Earth-collision geometries (two "branches" of the stream): the Earth is hit by particles on their first node crossing and by those on their second one (Baggaley \& Neslušan 2002). In the first case, grains strike the Earth before the perihelion passage, and in the second they first travel around the Sun and only then reach the Earth. This is illustrated in Fig. 3. Our calculations (see Eqs. (11)-(14) below) show that the dust flux in the second branch is lower than in the first by only a factor of 1.6 to 2.5 , depending on the season (the angle $\alpha$ in Fig. 3). We found, however, that particles of the second branch should arrive at Earth from geocentric declinations ranging from $+26^{\circ}$ (in northern winter) to $+44^{\circ}$ (in northern summer). Since AMOR, located at temperate southern latitudes, can cover the range $-90^{\circ}<$ $\delta<+20^{\circ}$ (Baggaley 2000), it only detects the first branch. Observations of the second one require a radar located in the equatorial region or in the northern hemisphere. If such an instrument is available in the future, a detection of the second branch will be a crucial test of $\beta$ Pic as the source of the dust particles.

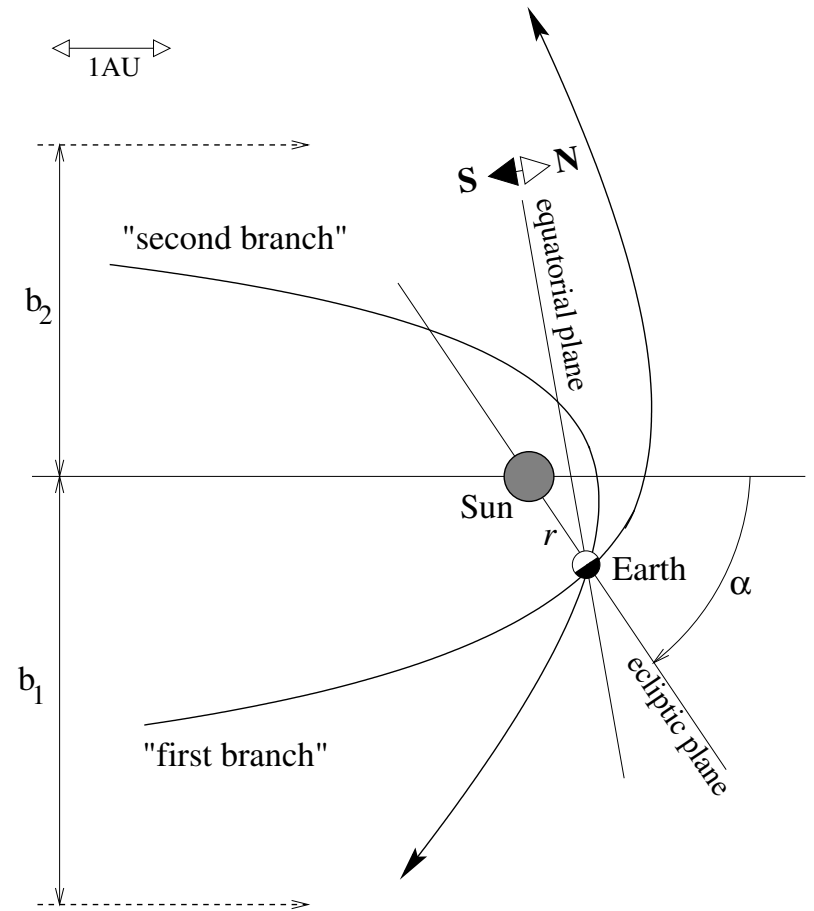

Fig. 3. Deflection of the meteoroid trajectories by the gravity of the Sun. The meteoroids are coming from the left. The Sun and Earth are not to scale. The position of the Earth shown corresponds to the beginning of January, shortly after a winter solstice (solar longitude of $280^{\circ}$ ). At this moment, $\alpha$ (the angle between the heliocentric radius vector of the Earth and the velocity vectors of the stream particles far from the Sun, depicted with dashed arrows) reaches a minimum possible value of $56^{\circ}$. Both ecliptic and equatorial planes are seen nearly edge-on, whereas the direction to the vernal equinox (not shown) is nearly perpendicular to the figure plane towards the reader. This geometry is determined by the far-Sun ecliptic latitude and longitude of the meteoroid source, $-56^{\circ}$ and $280^{\circ}$. The Earth is hit by two hyperbolic trajectories ("first branch" and "second branch"), coming to the Earth from nearly opposite directions. For the particular season shown, the impact parameters of the two trajectories are $b_{1}=3.8 \mathrm{AU}$ and $b_{2}=3.0 \mathrm{AU}$ and the perihelion distances are $0.94 \mathrm{AU}$ and $0.59 \mathrm{AU}$, respectively. Only the first branch can be observed from New Zealand which, for the season shown here, is also obvious from the figure.

\section{2. $\beta$ Pictoris system}

\section{Age of the star}

Earlier estimates proposed a wide range of possible ages from about 10 up to more than $300 \mathrm{Myr}$, before Barrado y Navascués et al. (1999) found two other stars comoving with $\beta$ Pic in space, allowing them to narrow the estimates to the values $20 \pm$ 10 Myr. Subsequently, the number of members of this Beta Pictoris Moving Group was increased to 28 stars and brown dwarfs by Zuckerman et al. (2001). The re-analysis of the BPMG evolutionary age by means of the H-R diagram yielded $12_{-4}^{+8}$ Myr. Recently, Ortega et al. (2002) retraced the 3D orbits of the group members in the Galaxy and found the maximum concentration to occur 11.5 Myr ago, which suggests this value to be the kinematic age of $\beta$ Pic and other members of the BPMG. 


\section{Possible planets}

A dozen asymmetries and individual features of different types have been identified on the disk images (Kalas \& Jewitt 1995; Kalas et al. 2000, 2001; Wahhaj et al. 2003). Whereas the clumps in the outermost part of the NE wing (500 to $800 \mathrm{AU}$ ) have been attributed to a recent stellar encounter (Kalas et al. 2000, 2001), many authors have invoked planets in the $\beta$ Pic system to explain a number of other intricate features observed in the disk. Artymowicz et al. (1989) and Lagage \& Pantin (1994) reported a brightness gap in the disk inside about 10 to $20 \mathrm{AU}$ from the star, which stimulated intensive discussions of an alleged planetary perturber that could clear up the inner region (Scholl et al. 1993; Lagage \& Pantin 1994; Roques et al. 1994; Lazzaro et al. 1994; Lecavelier des Etangs et al. 1996a; Liou \& Zook 1999). The basic idea is that the dust migrating towards the star due to the Poynting-Robertson effect is ejected out of the system during close encounters with planets, possibly after trapping into outer mean-motion resonances (MMRs) and subsequent eccentricity pumping. Warps of the inner disk (see, e.g., Mouillet et al. 1997; Heap et al. 2000) can also be attributed to the presence of a planet. Mouillet et al. (1997) inferred a planet in a slightly inclined orbit at about 3 to $20 \mathrm{AU}$ with a mass comparable to that of Jupiter, and Augereau et al. (2001) have shown that the same planet could account for the so-called butterfly asymmetry of the disk. Finally, recently imaged dust rings and "new" warps in the inner disk (Wahhaj et al. 2003; Weinberger et al. 2003) seem to directly indicate the presence of several planets between about 15 and $80 \mathrm{AU}$. To summarize, all these considerations indicate the presence of a few planets with semimajor axes in the range from several to several tens of AU. Nothing is known, however, about presence or absence of planets inside several AU from the star.

As noted by Beust \& Morbidelli (2000), all of the mechanisms proposed so far to explain the FEB phenomenon involve gravitational perturbations by at least one planet: close encounters (Beust et al. 1991), the Kozai mechanism (Bailey et al. 1992), trapping in MMRs. Some mechanisms, such as secular resonances, require more than one planet. See Beust \& Morbidelli (1996, 2000), Thébault \& Beust (2001) for a review of different mechanisms. The most advocated mechanism is an eccentricity pumping of a cometesimal trapped into a 4:1 or 3:1 MMR with a Jupiter-like planet in a moderatelyeccentric $\left(e_{\mathrm{p}} \gtrsim 0.05\right)$ orbit at 5 to $20 \mathrm{AU}$ from the star (Beust \& Morbidelli 1996, 2000; Quillen \& Holman 2000; Thébault $\&$ Beust 2001). It could, in particular, be the same planet as the one responsible for the "classical" warp (Thébault \& Beust 2001). The main difficulty of the mechanism is refilling the resonant locations with cometesimals. Either planet migration (Beust \& Morbidelli 1996, 2000) or collisions between the planetesimals (Thébault \& Beust 2001) could be a key.

\section{Cometesimals}

Little is known about size/mass and orbital distributions of small bodies in the disk and the dust material they produce. Attempts to constrain orbital distributions of dust grains from the observed brightness profiles give non-unique results

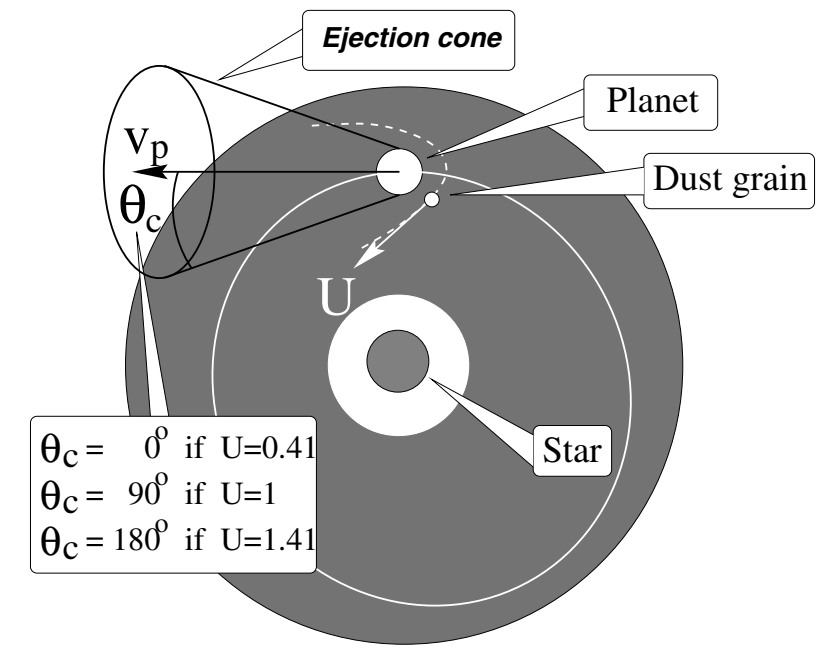

Fig. 4. Illustration of the geometry underlying Öpik's theory.

(Lecavelier des Etangs et al. 1996b). Theoretical approaches to clarify this question are hampered by a variety of counteracting dynamical processes that are hard to describe (mutual collisions, radiation pressure dynamics of particles with poorly known properties, resonant influence of planets with unknown masses and orbits, drag forces by a poorly known gas component). In particular, it is not known how large the typical eccentricities of both parent bodies and dust grains are. (Note a close relation between them: orbits of dust grains are essentially those of the parent bodies, somewhat modified by direct radiation pressure upon release from the sources, Burns et al. 1979.) It is difficult to decide whether the cometesimal disk and the disk of larger dust grains are rather "circular" or "eccentric". Most probably, the distribution of eccentricities is broad, including both near-circular and eccentric populations.

The same applies to the size distribution of dust. Observations suggest that grains in a broad size range from submicrometers to millimeters are present, but do not give information on the relative contribution of particles with different sizes (Krivova et al. 2000). Dynamical modeling confirms that the size distribution is broad and suggests several tens of micrometers as a typical size of grains, but should be considered with caution, because of a number of simplifying assumptions in the model and poorly known model parameters (Krivov et al. 2000).

\section{Planetary scenario}

\subsection{Semianalytic calculations}

One of the parameters of the stream most difficult to explain is the very high ejection speed of the stream particles. This places severe constraints on the mass and orbit of a planetary ejector. Prior to any calculations, it can be expected that sufficiently high ejection speeds can only be obtained in "violent" scenarios. To demonstrate this, we make use of Öpik's (1976) theory of planetary encounters.

The Öpik theory considers a star, a planet, and a massless particle (or a disk of non-interacting particles) initially placed in a planet-crossing orbit (Fig. 4). The basic quantity in the 
theory is the planetocentric grain velocity $\boldsymbol{U}$. In units of $\left\langle v_{\mathrm{p}}\right\rangle$, the averaged planet orbital velocity, $U$ is a dimensionless quantity. The idea is to assume each encounter of the particle with the planet to be an impulse in $\boldsymbol{U}$, so that $\boldsymbol{U}(t)$ is treated as a stochastic process. In the particular case of a circular planetary orbit, $U$ is related to the Tisserand constant $T: U=\sqrt{3-T}$. Therefore, $\sqrt{\left\langle U^{2}(t)\right\rangle}=$ const. For an elliptic planetary orbit, $\sqrt{\left\langle U^{2}(t)\right\rangle}$ grows with time (dynamical heating of the disk). Ejection out of the system becomes possible when $U$ grows to the value $\sqrt{2}-1 \approx 0.41$ and occurs if and only if $\boldsymbol{U}$ falls into the cone with an opening angle $\theta_{\mathrm{c}}(U)$ around $\boldsymbol{v}_{\mathrm{p}}$. When $U=1$, the probability that an encounter results in ejection is $1 / 2\left(\theta_{\mathrm{c}}(U)=90^{\circ}\right)$. Finally, for $U \geq \sqrt{2}+1 \approx 1.41$ ejection is the only possible outcome of any encounter with the planet $\left(\theta_{\mathrm{c}}(U)=180^{\circ}\right)$.

For each instant in time, the theory allows the calculation of $U$ and a number of other quantities of interest: the probability of ejection, that of collision with the planet, the rms speed of ejected particles at infinity, etc. For the algorithm, the reader is referred to Öpik's (1976) original book or, alternatively, to Farinella et al. (1990), where a concise description of the algorithm can be found.

Figure 5 depicts results of calculations that we made following Öpik for different parameters of the planetary perturber in the $\beta$ Pic disk. For the disk itself, we assumed initially circular orbits of grains with initial inclinations uniformly distributed within $\left[0^{\circ}, 7^{\circ}\right]$, in accordance with the observed halfopening angle of the disk of $7^{\circ}$. The panels from top to bottom show the rms encounter velocity, an upper limit on the speed of the ejected particles at infinity, and the probabilities of a particle's survival against ejection and collision with the planet. The columns from left to right illustrate the dependence of the results on the planet's semimajor axis, mass, and orbital eccentricity. Altogether, Öpik's theory predicts that faster ejecta require either large planetary masses, small semimajor axes, or substantial eccentricity of the planetary orbit. It is also seen that ejection is always more probable than collision with the planet.

As Öpik's theory is only a crude approximation (see, e.g., Weidenschilling 1975, for a detailed discussion), these results can only be used as guidelines for more accurate numerical simulations. These are described in the following section.

\subsection{Numerical simulations}

\section{Model}

We performed numerical integrations with (a slightly adapted) Duncan and Levison's SWIFT/SyMBA v2.0 package (Duncan et al. 1998). Cometesimals are initially placed in planetcrossing orbits to speed up the ejection outcome. Another reason to do this is that our model does not take into account processes with longer timescales (migration of planets, mutual collisions between cometesimals etc.).

\section{Initial data}

"Conventional" planet. As noted above, there is a considerable body of indirect evidence of the presence of planets in the $\beta$ Pic system. It was deduced from the analysis of disk's asymmetries, warps (see, e.g., Mouillet et al. 1997; Heap et al. 2000) as well as recently discovered dust rings and warps in the innermost part of the disk (Wahhaj et al. 2003; Weinberger et al. 2003). All these considerations suggest several planets with semimajor axes between several and almost a hundred AU. Note that symmetry of the isolated C- and D-dust features observed at 50 and $80 \mathrm{AU}$ (Wahhaj et al. 2003) seems to favor low eccentricities of the outer planets possibly located at these distances. The alleged planets closer to the star could be on eccentric orbits, however. In both cases, there appears to be no direct upper limit on the planetary masses. We will first check whether these "conventional" planets can be responsible for the observed stream.

Planet at an intermediate distance. The second idea is to "place" a planet yet closer to the star, at about $1 \mathrm{AU}$, i.e. at the outer edge of the region where many extrasolar planets have been discovered by radial velocity $(R V)$ measurements. The planets of this kind detected so far have substantial orbital eccentricities (Marcy \& Butler 2000), which makes them potentially efficient "ejectors" of material.

Close-in planet. Finally, we will consider "hot Jupiters", representing roughly half of all planets discovered so far. Such planets have a semimajor axis of several tenths of an AU. Their proximity to the star would make material ejection quite efficient. However, this is counteracted somewhat by the fact that these close-in companions all have small eccentricities $e_{\mathrm{p}} \lesssim 0.1$ (Marcy \& Butler 2000), which is unfavorable for high-speed ejection. What is more, such a planet cannot be too massive in the case of $\beta$ Pic, otherwise it would be evident in $R V$ measurements. For FGKM stars usually searched with the $R V$ method, the "threshold" $R V$ amplitude is $\sim 10 \mathrm{~m} \mathrm{~s}^{-1}$ (see, e.g., Ksanfomaliti 2000, and references therein). For the $\beta$ Pictoris mass (but assuming slow rotation of the star and no appreciable photospheric jitter, similar to main-sequence FGKM stars), the maximum nondetectable planet's mass $m_{\mathrm{p}}$ is related to its orbital semimajor axis $a_{\mathrm{p}}$ as:

$m_{\mathrm{p}}\left[M_{\mathrm{J}}\right] \approx 0.5 \sqrt{a_{\mathrm{p}}[\mathrm{AU}]}$.

However, $\beta$ Pictoris is an $\mathrm{A} 3 \mathrm{~V}$ star, so the threshold must be appreciably higher: Mouillet et al. (1997), for instance, refer to threshold values as large as $1000 \mathrm{~m} \mathrm{~s}^{-1}$, increasing the factor in (4) from 0.5 to 50 . In what follows, we assume that a Jupitermass planet at $0.1 \mathrm{AU}$ from the star would not show up in any $R V$ searches.

\section{Results}

Typical results are collected in Table 3. The left two columns are for the conventional planet case, the middle two for planets at intermediate distance, and the right two ones represent closein planets.

The conventional planets can hardly account for the highspeed ejection, even if the planetary orbit has a substantial eccentricity, the planet is massive, and the cometesimal disk is dynamically hot. The same applies to planets at 1 AU. Speeds of several $\mathrm{km} \mathrm{s}^{-1}$ are the maximum possible in these cases. 


$$
a_{p}=^{*}, m_{p}=10, e_{p}=0.4
$$
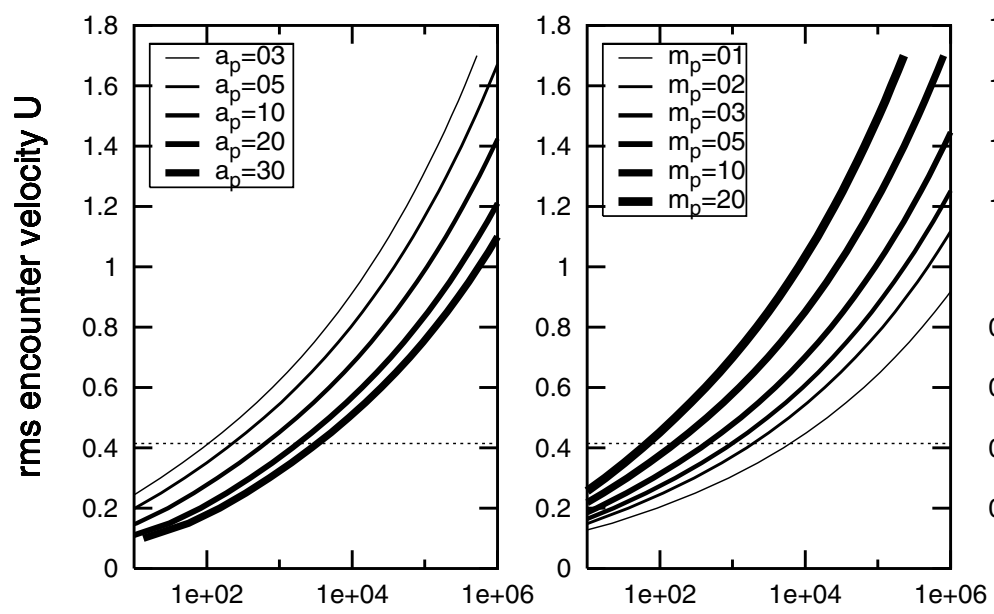

$a_{p}=04, m_{p}=20, e_{p}=^{*}$
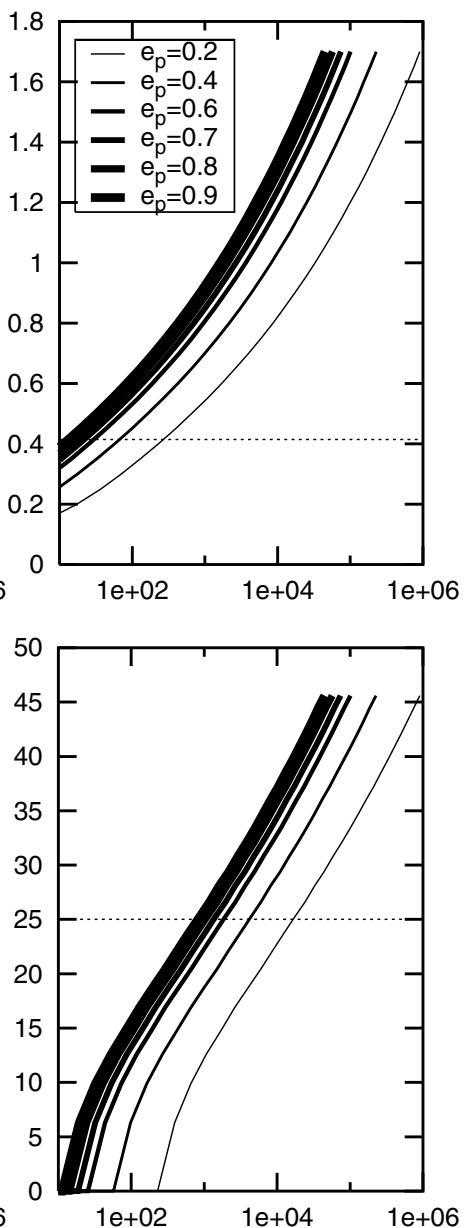
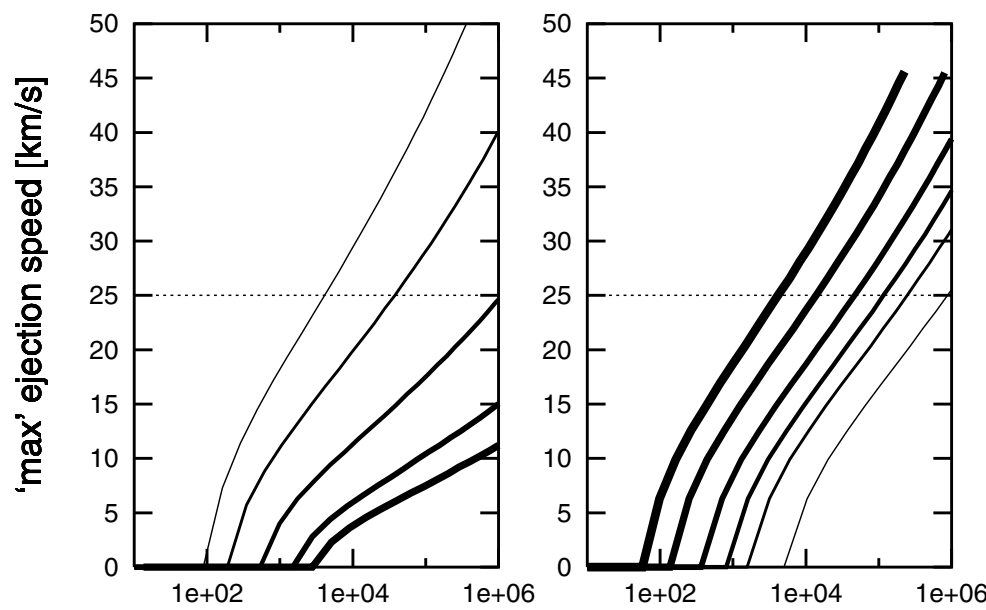

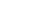
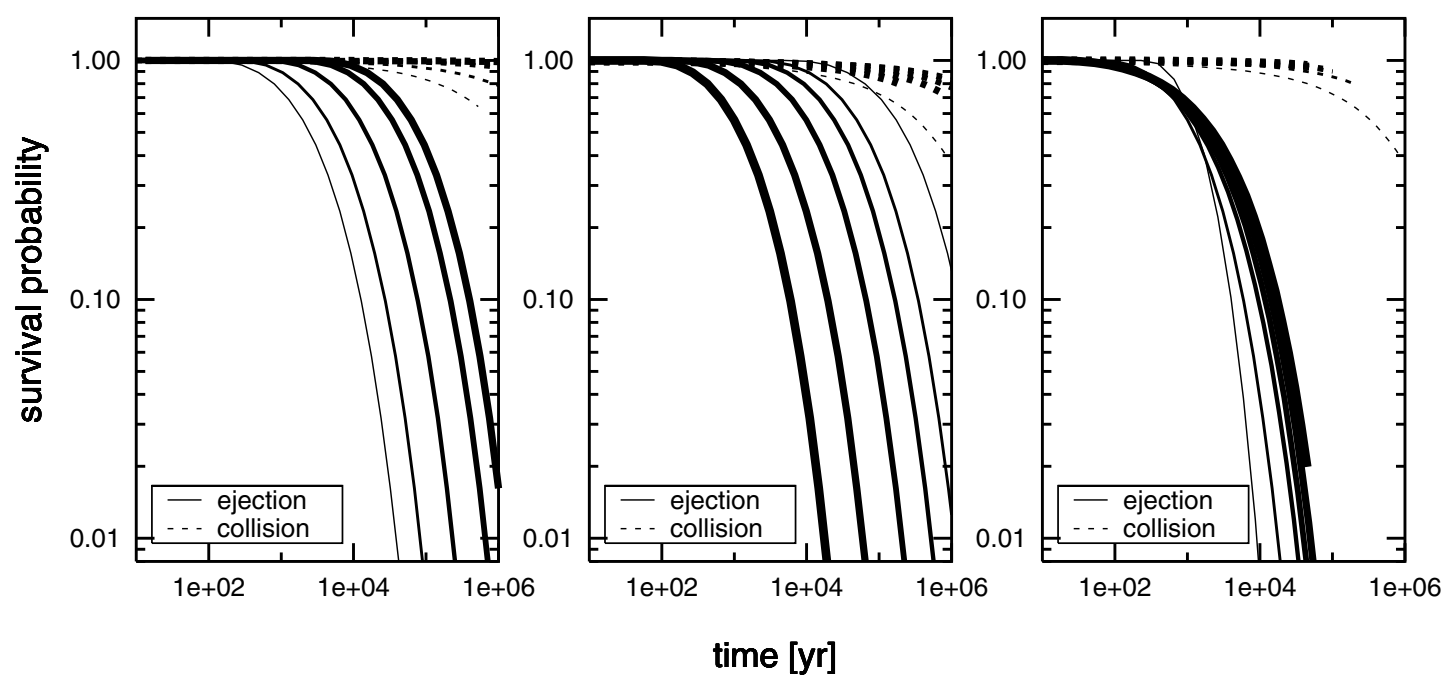

Fig. 5. Results obtained with Öpik's theory for different parameters of a hypothetical planet in the $\beta$ Pic disk. Top row: rms encounter velocity, middle row: upper limits on the speed of the ejected particles at infinity, bottom row: the probability of a particle's survival against ejection (solid) and collision with the planet (dashed). Left column: dependence on planet's semimajor axis $a_{\mathrm{p}}$ (its mass and eccentricity remain fixed), middle column: dependence on planet's mass $m_{\mathrm{p}}$ (semimajor axis and eccentricity fixed), right column: dependence on planet's eccentricity $e_{\mathrm{p}}$ (its semimajor axis and mass fixed). See legend for adopted parameter values (semimajor axis in AU, mass in Jupiter masses). The critical value of $U$ for ejection is indicated by the dotted line in the top panels. The dotted line in the middle panels depicts the ejection speed of $25 \mathrm{~km} \mathrm{~s}^{-1}$ inferred from the radar measurements. 
Table 3. Outcome of the numerical runs. Columns from left to right: distant massive planet in near-circular orbit, initially cold disk (in the dynamical sense); distant massive planet in eccentric orbit, initially hot disk; Jupiter-mass planet in eccentric orbit at intermediate distance, hot disk; massive planet in eccentric orbit at intermediate distance, hot disk; close-in Neptune-mass planet in near-circular orbit, very hot disk; close-in Jupiter-mass planet in near-circular orbit, very hot disk. Initial inclination distribution: from within $\left[0^{\circ}, 7^{\circ}\right]$ ("wedged disk"). Integration interval: 30000 years. Number of particles in each run: 1000. All percentages are with respect to this number.

\begin{tabular}{|c|c|c|c|c|c|c|}
\hline Planet & $\begin{array}{r}m_{\mathrm{p}}=10 M_{\mathrm{J}} \\
a_{\mathrm{p}}=10 \mathrm{AU} \\
e_{\mathrm{p}}=0.1\end{array}$ & $\begin{array}{r}m_{\mathrm{p}}=10 M_{\mathrm{J}} \\
a_{\mathrm{p}}=10 \mathrm{AU} \\
e_{\mathrm{p}}=0.7\end{array}$ & $\begin{array}{r}m_{\mathrm{p}}=1 M_{\mathrm{J}} \\
a_{\mathrm{p}}=1 \mathrm{AU} \\
e_{\mathrm{p}}=0.4\end{array}$ & $\begin{array}{r}m_{\mathrm{p}}=5 M_{\mathrm{J}} \\
a_{\mathrm{p}}=1 \mathrm{AU} \\
e_{\mathrm{p}}=0.4\end{array}$ & $\begin{array}{r}m_{\mathrm{p}}=0.1 M_{\mathrm{J}} \\
a_{\mathrm{p}}=0.1 \mathrm{AU} \\
e_{\mathrm{p}}=0.0\end{array}$ & $\begin{array}{r}m_{\mathrm{p}}=1 M_{\mathrm{J}} \\
a_{\mathrm{p}}=0.1 \mathrm{AU} \\
e_{\mathrm{p}}=0.0\end{array}$ \\
\hline \multirow{3}{*}{ Disk } & $a=10 \mathrm{AU}$ & $a=10 \mathrm{AU}$ & $a=10 \mathrm{AU}$ & $a=10 \mathrm{AU}$ & $a=10 \mathrm{AU}$ & $a=10 \mathrm{AU}$ \\
\hline & $e_{\min }=0.0$ & $e_{\min }=0.0$ & $e_{\min }=0.9$ & $e_{\min }=0.9$ & $e_{\min }=0.99$ & $e_{\min }=0.99$ \\
\hline & $e_{\max }=0.1$ & $e_{\max }=0.99$ & $e_{\max }=0.99$ & $e_{\max }=0.99$ & $e_{\max }=0.999$ & $e_{\max }=0.999$ \\
\hline \multicolumn{7}{|c|}{ Loss Statistics } \\
\hline e-folding lifetime (63\% lost), yr & $\gtrsim 3 \times 10^{4}$ & $\gg 3 \times 10^{4}$ & $3 \times 10^{4}$ & $3 \times 10^{3}$ & $6 \times 10^{3}$ & $7 \times 10^{2}$ \\
\hline Collided with star, $\%$ & 1 & 27 & 15 & 25 & 1 & 9 \\
\hline Collided with planet, $\%$ & 1 & 0 & 1 & 1 & 16 & 5 \\
\hline Stuck at the periphery, $\%$ & 16 & 4 & 9 & 6 & 8 & 3 \\
\hline Ejected, $\%$ & 41 & 19 & 45 & 66 & 73 & 83 \\
\hline Total, \% & 59 & 50 & 70 & 98 & 97 & 100 \\
\hline \multicolumn{7}{|c|}{ Ejection Speed } \\
\hline Mean, $\mathrm{km} \mathrm{s}^{-1}$ & 0.6 & 1.1 & 1.3 & 1.5 & 2.1 & 3.9 \\
\hline Max, $\mathrm{km} \mathrm{s}^{-1}$ & 1 & 5 & 8 & 9 & 66 & 94 \\
\hline Fraction $>25 \mathrm{~km} \mathrm{~s}^{-1}, \%$ & 0 & 0 & 0 & 0 & 0.3 & 2 \\
\hline
\end{tabular}

On the other hand, the close-in planet case seems to work quite well, ejecting the planet-crossing solids rapidly and at high speeds. Several percent of all particles were ejected at speeds of tens $\mathrm{km} \mathrm{s}^{-1}$.

A substantial fraction of the material, especially in less "violent" scenarios, only reaches the periphery of the system, where it remains ("Oort cloud formation"). Also, an appreciable amount of star-grazers (several to several tens of percent) is always produced. In contrast, collisions with a planet are rare (several percent at most).

A particle placed in a hyperbolic trajectory can, in principle, be lost by grain-grain collisions before it can leave the system. Simple estimates based on collisional probabilities (cf. Krivov et al. 2000) show that this effect is statistically unimportant. A rather low upper limit on the molecular hydrogen contents in the disks recently obtained (Lecavelier des Etangs et al. 2001) shows that the gas drag force is not important either.

\section{Radiation pressure scenario}

To explain the dust stream phenomenon, Grün \& Landgraf (2001) have proposed a radiation pressure ejection of dust material released from cometesimals. The same mechanism has long been known to work in the solar system, accounting for the so-called $\beta$-meteoroids in interplanetary space (Zook \& Berg 1975). The $\beta$-meteoroids in the solar system are thought to be produced mostly by catastrophic fragmentation of larger interplanetary dust particles (IDPs). Tiny submicrometer-sized grains produced by these collisions can easily get into escape orbits even from initially circular orbits of the source IDPs.

In contrast, the stream meteoroids detected by radar measurements are large $(\gtrsim 20 \mu \mathrm{m})$, so that the radiation pressure to gravity ratio $\beta \ll 1$. This makes a hyperbolic ejection only

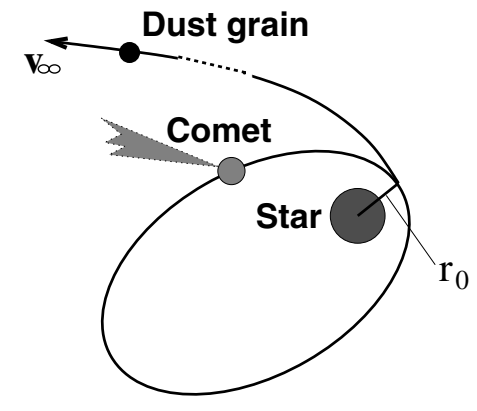

Fig. 6. Illustration of ejection by radiation pressure.

possible near periastra of cometesimals, and only if these are moving in highly eccentric orbits (Fig. 6).

We now estimate the condition for the ejection of these extrasolar $\beta$-meteoroids and the asymptotic speed. Consider a dust grain with a certain $\beta$-ratio $0<\beta<1$ (the case $\beta \geq 1$ is not of interest, because it applies for too small particles). Let the grain be ejected from a parent body that moves in an elliptic orbit with eccentricity $0 \leq e<1$. Assume that the ejection takes place at the pericenter of the parent body orbit. Denote the distance of this point to the star by $r_{0}$. The grain will be ejected out of the system (in a hyperbolic orbit) if and only if:

$2 \beta+e-1>0$

Denoting by $v_{0}$ the circular Keplerian speed at the ejection distance $r_{0}$, the grain speed at infinity will be:

$v_{\infty}=v_{0} \sqrt{2 \beta+e-1}$ 
Now, restricting ourselves to the case of high eccentricities, $e \lesssim$ 1, we get a final estimate:

$v_{\infty} \lesssim v_{0} \sqrt{2 \beta}$.

For $\beta$ Pic,

$v_{0}=40 \mathrm{~km} \mathrm{~s}^{-1}\left(\frac{r_{0}}{1 \mathrm{AU}}\right)^{-1 / 2}$.

For grains with radii of 20 to $30 \mu \mathrm{m}$, plausible materials and grain compositions give $\beta$ values between about 0.05 and 0.35 (Krivov et al. 1998). Here we have multiplied the $\beta$ values, given in that paper for the solar system dust, by $L_{\star} / M_{\star}=$ $8.70 / 1.75=5.0$, where $L_{\star}$ and $M_{\star}$ are stellar luminosity and mass, respectively, both in solar units. Such grains are ejected at a speed

$v_{\infty} \lesssim 13$ to $33 \mathrm{~km} \mathrm{~s}^{-1}\left(\frac{\mathrm{r}_{0}}{1 \mathrm{AU}}\right)^{-1 / 2}$.

Thus the radiation pressure mechanism can explain the stream, easily providing values $\gtrsim 25 \mathrm{~km} \mathrm{~s}^{-1}$ when ejection occurs from several tenths of an AU. We stress that high eccentricities are imperative for the radiation pressure mechanism to work. Equation (6) shows that for $e<0.3$ to 0.9 (depending on the $\beta$ value adopted), ejection by radiation pressure is not possible at all.

\section{Detected dust flux and mass loss rate from the disk}

The estimate of the absolute dust flux at Earth quoted in Sect. $2.1,3 \times 10^{-17} \mathrm{~cm}^{-2} \mathrm{~s}^{-1}$, allows estimation of the mass loss rate from the $\beta$ Pic system.

First, one has to correct the flux for the gravitational focusing by the Sun (a similar effect caused by the Earth is unimportant). Consider a point with the radius vector $r$ and let $\alpha$ be the angle between $\boldsymbol{r}$ and the direction of the stream (Fig. 3). The number density of the focused dust at this point is given by (Fessenkov 1947; Fahr 1968):

$n(r, \alpha)=n_{1}(r, \alpha)+n_{2}(r, \alpha)$,

where:

$\frac{n_{1,2}}{n_{\infty}}=\frac{b_{1,2}^{2}}{r \sin \alpha\left|2 b_{1,2}-r \sin \alpha\right|}$,

and

$b_{1,2}=\frac{r \sin \alpha}{2} \pm \sqrt{\left(\frac{r \sin \alpha}{2}\right)^{2}+\frac{G M r(1+\cos \alpha)}{v_{\infty}^{2}}}$.

Here $n_{\infty}$ and $v_{\infty}$ are respectively the number density and velocity (with respect to the Sun) of the far-Sun meteoroids and $G M$ is the gravitational parameter of the Sun. The indices 1, 2 in the above equations correspond to the first and second "branches", discussed in Sect. 2.1 and shown in Fig. 3.

Not only does the gravity of the Sun increase the number density, it also increases the speed of meteoroids. At the same point with the radius vector $\boldsymbol{r}$, the speed is

$v(r)=\sqrt{v_{\mathrm{esc}}^{2}+v_{\infty}^{2}}$, where $v_{\text {esc }}=\sqrt{2 G M / r}$ is the escape velocity at the distance $r$. The relation between the dust fluxes at the point $(r, \alpha)$ and at infinity is obviously

$\frac{F(r, \alpha)}{F_{\infty}}=\frac{n(r, \alpha)}{n_{\infty}} \frac{v(r)}{v_{\infty}}$.

Given the ecliptic latitude of the meteoroid stream far from the Sun of $-56^{\circ}$, deduced from the AMOR observations, the angle $\alpha$ varies between $56^{\circ}$ and $180^{\circ}-56^{\circ}=124^{\circ}$, as the Earth orbits the Sun. The distance $r=1$ AU. Taking into account the first branch only (the second one is not detectable with AMOR, see Sect. 2.1), Eq. (14) gives

$\frac{F}{F_{\infty}}=6$ to 10 .

Thus, the flux entering the solar system is approximately $\sim 3 \times$ $10^{-18} \mathrm{~cm}^{-2} \mathrm{~s}^{-1}$.

Next, we correct the flux for the relative velocity between $\beta$ Pic and the Sun. With the ejection speed from $\beta$ Pic of $\approx 25 \mathrm{~km} \mathrm{~s}^{-1}$ and the heliocentric speed of the grains entering the solar system of $\approx 11 \mathrm{~km} \mathrm{~s}^{-1}$ (Table 2), the corrected flux is $25 / 11 \times 3 \times 10^{-18} \mathrm{~cm}^{-2} \mathrm{~s}^{-1} \approx 7 \times 10^{-18} \mathrm{~cm}^{-2} \mathrm{~s}^{-1}$.

Finally, we assume a standard "wedged" $\beta$ Pic disk with a half-opening angle of $\sim 0.1$ radian seen edge-on and a distance to the star of $19.3 \mathrm{pc}$ to get the mass loss rate in the form of $10^{-7} \mathrm{~g}$-mass meteoroids (the AMOR threshold) of $\sim 2 \times 10^{-5} M_{\oplus} \mathrm{yr}^{-1}$.

This is probably an underestimate of the total loss rate, because it implies that all the material is lost in the form of $10^{-7}$ g-mass (or $\sim 20 \mu \mathrm{m}$-sized) dust grains, as detected at Earth. And here is perhaps the most uncertain part of the whole estimation: one has to invoke a mass/size distribution in the disk, which is actually unknown. Assuming a two-slope distribution with the mass distribution index $1 / 3$ for grains $<10^{5} \mathrm{~g}$ and $4 / 3$ for more massive ones, as suggested by the modeling (Krivov et al. 2000), we find that the total mass loss rate from the disk is about 40 times the mass loss rate in the form of grains with masses of $10^{-7} \mathrm{~g}$, or $\sim 7 \times 10^{-4} M_{\oplus} \mathrm{yr}^{-1}$.

One can speculate, however, that the ejection mechanism can be strongly selective about the particle masses. For instance, the radiation pressure only ejects grains smaller than several tens of $\mu \mathrm{m}$, so the larger solids would stay in the disk. This would mean that most of the mass is lost in the form of ten- $\mu \mathrm{m}$ grains, which is coincidentally the detection range of the radar. Even for size-independent mechanisms, such as the planetary ejection, it may be that larger solids could not leave the system - for example, they could have been destroyed by collisions before they could leave, as suggested by Stern \& Weissman (2001) for the Oort cloud formation phase in the early solar system. Thus, the "uncorrected" estimate, $\sim 2 \times 10^{-5} M_{\oplus} \mathrm{yr}^{-1}$, could be close to the total mass loss rate. We are left with a range from $\sim 2 \times 10^{-5} M_{\oplus} \mathrm{yr}^{-1}$ up to $\sim 7 \times 10^{-4} M_{\oplus} \mathrm{yr}^{-1}$.

Similar to the correction for a mass distribution, a correction for a speed distribution of the particles has to be made. Namely, the total mass loss rate derived above must be divided by a fraction of ejected grains whose ejection speeds are high enough to satisfy the kinematic conditions, $\approx 25 \mathrm{~km} \mathrm{~s}^{-1}$. 
However, this fraction cannot be estimated in any reliable way for both planetary and radiation pressure ejection scenarios. In the planetary ejection scenario, it could be $\sim 1 \%$ as suggested by the numerical runs (see Table 3), but could be quite different for different parameters of the planet and the disk. In the radiation pressure ejection scenario, the fraction in question is largely controlled by the ejection distance $r_{0}$ (see Eq. (9)), which is not known either. Asserting, somewhat arbitrarily, the fraction to be $0.01-0.1$, we finally get the mass loss rate in the range from $\sim 2 \times 10^{-4} M_{\oplus} \mathrm{yr}^{-1}$ to $\sim 7 \times 10^{-2} M_{\oplus} \mathrm{yr}^{-1}$.

This immediately shows that the ejection process could not take place on timescales comparable with the stellar age: the mass loss over $10^{7} \mathrm{yr}$ would be $2 \times 10^{3}$ to $7 \times 10^{5} M_{\oplus}$, i.e. comparable to, or more than a primordial $\beta$ Pic protoplanetary disk could have contained. We note that such a long timescale is already refuted by stream geometry arguments: as we saw in Sect. 2.1, the ejection process started working not earlier than $\approx 8.5 \times 10^{5} \mathrm{yr}$ ago. The resulting mass loss, reduced by an order of magnitude, still appears quite large. Krivova \& Solanki (2003) suggested to mitigate the problem by assuming that the process took place during a recent intensive clearance phase of the primordial disk, with a probable duration of $\sim 10^{5} \mathrm{yr}$, and that we are currently observing the system soon after this phase took place. The low age of $\beta$ Pic, a high dustiness of its disk, as well as the low hydrogen contents insufficient for a Jupiter-like planet formation are all in favor of the idea, suggesting that the system is, or recently was, at a fairly advanced planetary accretion phase. With an ejection phase of that duration, the total mass loss could be as low as $20 M_{\oplus}$. Quantitatively, such a value is also consistent with the likely total mass of the primordial disk of $\lesssim 10^{4} M_{\oplus}$ (assuming a disk radius of $\lesssim 10^{3} \mathrm{AU}$ and a linear density of $10 M_{\oplus} \mathrm{AU}^{-1}$, see Thébault \& Beust 2001).

Finally, as we have shown here, the only "channel" for high-speed ejection of material (in both scenarios, planetary ejection and radiation pressure blowout) is active in close vicinity of the star, $\lesssim 1 \mathrm{AU}$. In other words, a sufficient fraction of the primordial disk mass had to be placed, by whatever processes, into FEB-like orbits before it got ejected into interstellar space. This still appears to be realistic, but only if the mass loss rate was close to the lower limit, $\sim 10^{-4} M_{\oplus} \mathrm{yr}^{-1}$. Provided this is the case, the theoretical scenarios can account for both the deduced ejection speed and the observed flux at Earth.

\section{Conclusions}

1. Our stellar and dust kinematics calculations confirm an earlier conclusion that the $\beta$ Pictoris system is the likely source of the stream of interstellar meteoroids observed with the AMOR radar (Baggaley 2000). We find that the dust grains must have been ejected from $\beta$ Pic about $0.7 \mathrm{Myr}$ ago at speeds $\sim 25 \mathrm{~km} \mathrm{~s}^{-1}$. Further observational tests to prove that $\beta$ Pic is the source of the stream are pointed out.

2. Ejection speeds can be explained by both planetary ejection and radiation pressure mechanisms, putting the following constraints:

(a) A planet or planets at about one to several tens of AU from the star cannot be directly responsible for the stream, even if they are very massive and move in quite eccentric orbits. However, the same scenario works quite well with a possible "hot Jupiter" in the system - a Neptune- or Jupiter-mass companion in a near-circular orbit at a fraction of an AU from the star.

(b) The radiation pressure mechanism can also explain the stream, provided that most of the ejection occurs from (nearly) star-grazing comets and quite close to the star, at a fraction of an $\mathrm{AU}$.

(c) Both mechanisms do not rule out and can even amplify each other. For instance, even a terrestrial close-in planet could enhance the amount of material ejected by the radiation pressure, as well as the ejection speeds.

On any account, dust had to be ejected from the close ( $\lesssim 1$ AU) vicinity of the star and from orbits with high eccentricities. Evidence that the system does contain a population of comets (and therefore dust grains) in eccentric orbits comes from the observed FEB phenomenon. It is some of these comets, those with especially high orbital eccentricities and with favorable orientation of the orbits, that may cause the FEB events. It would be interesting to compare our estimates of the mass loss rate with those derived from the observed FEB statistics (frequencies of the events, estimates of probable mass loss per comet per periastron passage etc.). Unfortunately, such a quantitative analysis is not possible, because the FEB events are observed now and not $\sim 1$ Myr ago when the stream particles were ejected and when the system was most likely in a different state than at present.

3. The best current estimates of the detected dust flux at Earth are consistent with both scenarios as well, if we assume that the observed stream resulted from a recent intensive and short-lasting ( $\sim 0.1 \mathrm{Myr})$ clearance phase of $\beta$ Pic's protoplanetary disk and that the mass and speed distributions of the ejected solids are "favorable". The latter means that at least a percent or so of the ejected mass was accounted for by grains several tens of micrometers in size, having ejection speeds $\gtrsim 25 \mathrm{~km} \mathrm{~s}^{-1}$. In this respect, the radiation pressure ejection can be somewhat favored to the ejection by a close-in planet (more distant planets would fail to explain the high ejection speeds), because it is size-selective and easily yields higher ejection speeds. We stress, however, that the radiation pressure scenario also requires a massive planet in the system to supply a population of dust parent bodies in eccentric orbits.

4. Irrespective of the actual mechanism, the existence of the stream provides additional evidence that (i) a planet or planets exist in the $\beta$ Pic system and that (ii) the $\beta$ Pic disk has recently passed through an intensive "clearance stage".

Acknowledgements. We are most grateful to Jack Baggaley for numerous stimulating discussions and for commenting on earlier versions of the manuscript, and to Markus Landgraf for a speedy and thorough review. It is our pleasure to thank Sho Sasaki who attracted our attention to an "aberration" effect discussed in Sect. 2.1. Useful discussions with Amara Graps, Eberhard Grün, Hal Levison, Alessandro Morbidelli, Frank Spahn and Miodrag Sremčević are appreciated. 
We used a distribution of the SWIFT package from Hal Levison's web page, http://www.boulder.swri.edu/ ${ }^{\text {hal }}$. Miodrag Sremčević generously helped us with visualization of results presented in Fig. 2.

\section{References}

Artymowicz, P. 1997, Ann. Rev. Earth Planet. Sci., 25, 175

Artymowicz, P., Burrows, C., \& Paresce, F. 1989, ApJ, 337, 494

Augereau, J. C., Nelson, R. P., Lagrange, A.-M., et al. 2001, A\&A, 370,447

Baggaley, W. J. 2000, J. Geophys. Res., 105, 10353

Baggaley, W. J., \& Galligan, D. P. 2001, in Proc. of the Meteoroids Conf., 6-10 August 2001, Kiruna, Sweden, ESA SP-495, ed. B. Warmbein (Noordwijk: ESA Publications Division), 663

Baggaley, W. J., \& Neslušan, L. 2002, A\&A, 382, 1118

Bailey, M. E., Chambers, J. E., \& Hahn, G. 1992, A\&A, 257

Barrado y Navascués, D., Stauffer, J. R., Song, I., \& Caillault, J.-P. 1999, ApJ, 520, L123

Beust, H., Lagrange, A.-M., Plazy, F., \& Mouillet, D. 1996, A\&A, 310,181

Beust, H., Lagrange-Henri, A.-M., Vidal-Madjar, A., \& Ferlet, R. 1989, A\&A, 223, 304

Beust, H., \& Morbidelli, A. 1996, Icarus, 120, 358

Beust, H., \& Morbidelli, A. 2000, Icarus, 143, 170

Beust, H., Vidal-Madjar, A., \& Ferlet, R. 1991, A\&A, 247, 505

Blackwell, D. E., Leggett, S. K., Petford, A. D., Mountain, C. M., \& Selby, M. J. 1983, MNRAS, 205, 897

Burns, J. A., Lamy, P. L., \& Soter, S. 1979, Icarus, 40, 1

Crifo, F., Vidal-Madjar, A., Lallement, R., et al. 1997, A\&A, 320, L29

Duncan, M. J., Levison, H. F., \& Lee, M. H. 1998, AJ, 116, 2067

Fahr, H. J. 1968, Ap\&SS, 2, 474

Farinella, P., Paolicchi, P., Strom, R. G., \& Kargel, J. S. 1990, Icarus, 83, 186

Fessenkov, V. G. 1947, Meteoric matter in interplanetary space (Moscow-Leningrad: Academy of Sciences of the USSR), 276

Grün, E., \& Landgraf, M. 2001, Space Sci. Rev., 99, 151

Harvey, P. M., Wilking, B. A., \& Joy, M. 1984, Nature, 307, 441

Heap, S. R., Lindler, D. J., Lanz, T. M., et al. 2000, ApJ, 539, 435

Kalas, P., Deltorn, J.-M., \& Larwood, J. 2001, ApJ, 553, 410

Kalas, P., \& Jewitt, D. 1995, AJ, 110, 794

Kalas, P., Larwood, J., Smith, B. A., \& Schultz, A. 2000, ApJ, 530, L133

Krivov, A. V., Kimura, H., \& Mann, I. 1998, Icarus, 134, 311

Krivov, A. V., Mann, I., \& Krivova, N. A. 2000, A\&A, 362, 1127
Krivova, N. A. 2002, in Dust in the Solar System and Other Planetary Systems (COSPAR Coll. Ser. 15), ed. S. F. Green, I. P. Williams, J. A. M. McDonnell, \& N. McBride (Pergamon), 201

Krivova, N. A., Krivov, A. V., \& Mann, I. 2000, ApJ, 539, 424

Krivova, N. A., \& Solanki, S. K. 2003, A\&A, 402, L5

Ksanfomaliti, L. V. 2000, Sol. Sys. Res., 34, 481

Lagage, P. O., \& Pantin, E. 1994, Nature, 369, 628

Lagrange, A.-M., Ferlet, R., \& Vidal-Madjar, A. 1987, A\&A, 173, 289

Lazzaro, D., Sicardy, B., Roques, F., \& Greenberg, R. 1994, Icarus, 108,59

Lecavelier des Etangs, A. 2000, in Disks, Planetesimals and Planets, ed. F. Garzón, C. Eiroa, D. de Winter, \& T. J. Mahoney, ASP Conf. Ser., 219, 308

Lecavelier des Etangs, A., Scholl, H., Roques, F., et al. 1996a, Icarus, 123,168

Lecavelier des Etangs, A., Vidal-Madjar, A., \& Ferlet, R. 1996b, A\&A, 307, 542

Lecavelier des Etangs, A., Vidal-Madjar, A., Roberge, A., et al. 2001, Nature, 412, 706

Liou, J. C., \& Zook, H. A. 1999, AJ, 118, 580

Marcy, G. W., \& Butler, R. P. 2000, PASP, 112, 137

Mayor, M., \& Queloz, D. 1995, Nature, 378, 355

Mouillet, D., Larwood, J. D., Papaloizou, J. B., \& Lagrange, A.-M. 1997, MNRAS, 292, 896

Öpik, E. J. 1976, Interplanetary Encounters: Close Range Gravitational Interactions (New York, Elsevier)

Ortega, V. G., de la Reza, R., Jilinski, E., \& Bazzanella, B. 2002, ApJ, 575, L75

Quillen, A. C., \& Holman, M. 2000, AJ, 119, 397

Roques, F., Scholl, H., Sicardy, B., \& Smith, B. A. 1994, Icarus, 108, 37

Scholl, H., Roques, F., \& Sicardy, B. 1993, Celest. Mech. Dynam. Astron., 56, 381

Smith, B. A., \& Terrile, R. I. 1984, Science, 226, 1421

Stern, S. A., \& Weissman, P. R. 2001, Nature, 409, 589

Thébault, P., \& Beust, H. 2001, A\&A, 376, 621

Wahhaj, Z., Koerner, D. W., Ressler, M. E., et al. 2003, ApJ, 584, L27

Weidenschilling, S. J. 1975, AJ, 80, 145

Weinberger, A. J., Becklin, E. E., \& Zuckerman, B. 2003, ApJ, 584, L33

Weissman, P. R. 1984, Science, 224, 987

Zook, H. A., \& Berg, O. E. 1975, Planet. Space Sci., 23, 183

Zuckerman, B., Song, I., Bessell, M. S., \& Webb, R. A. 2001, ApJ, 562, L87 